\title{
A computational model of cuneothalamic projection neurons
}

\author{
Eduardo Sánchez ${ }^{1,3}$, Senén Barro ${ }^{1}$, Jorge Mariño ${ }^{2}$ and Antonio Canedo ${ }^{2}$ \\ ${ }^{1}$ Grupo de Sistemas Intelixentes (GSI), Departamento de Electrónica e Computación, Facultade \\ de Físicas, Universidade de Santiago de Compostela, 15706 Santiago de Compostela, Spain \\ ${ }^{2}$ Departamento de Fisioloxía, Facultade de Medicina, Universidade de Santiago de Compostela, \\ 15706 Santiago de Compostela, Spain \\ E-mail: eduardos@usc.es
}

Received 2 August 2001, in final form 2 September 2002

Published 7 February 2003

Online at stacks.iop.org/Network/14/211

\begin{abstract}
The dorsal column nuclei, cuneatus and gracilis, play a fundamental role in the processing and integration of somesthetic ascending information. Intracellular and patch-clamp recordings obtained in cat in vivo have shown that cuneothalamic projection neurons present two modes of activity: oscillatory and tonic (Canedo et al 1998 Neuroscience 84 603-17). The former is the basis of generating, in sleep and anaesthetized states, slow, delta and spindle rhythms under the control of the cerebral cortex (Mariño et al 2000 Neuroscience 95 657-73). The latter is needed, during wakefulness, to process somesthetic information in real time. To study this behaviour we have developed the first realistic computational model of the cuneothalamic projection neurons. The modelling was guided by experimental recordings, which suggest the existence of hyperpolarization-activated inward currents, transient low- and high-threshold calcium currents, and calcium-activated potassium currents. The neuronal responses were simulated during (1) sleep, (2) transition from sleep to wakefulness and (3) wakefulness under both excitatory and inhibitory synaptic input. In wakefulness the model predicts a set of synaptically driven firing modes that could be associated with information processing strategies in the middle cuneate nucleus.
\end{abstract}

\section{Introduction}

The cuneate nucleus $(\mathrm{CN})$ is a structure within the brainstem, close to the most rostral area of the spinal chord, where three different areas are distinguished: caudal, middle and rostral [3]. The

3 Author to whom any correspondence should be addressed. 
middle zone, where the experimental work reported in this study has been carried out, can in turn be divided into two sub-areas [13]: a core (central region), and a shell (peripheral region). The core comprises the dorsal region, and is basically made up of clusters of cuneothalamic neurons (projection or relay neurons), surrounded by local neurons (interneurons). The shell comprises the ventral and marginal regions, and is basically made up of interneurons.

The main cuneate inputs originate from primary afferent (PAF) and corticocuneate fibres. The PAFs establish synaptic contact with projection cells and interneurons [13]. The cortical fibres from the contralateral sensorimotor cortex run in the pyramidal tract $[34,39]$ and, within the middle cuneate, make synaptic contact mostly with inhibitory interneurons located in the shell [8]. On the other hand, the great majority of the projection neurons in the middle cuneate send their axons to the contralateral ventroposterolateral thalamic (VPL) nucleus via the medial lemniscus $[3,13,30]$.

To study the neuronal properties of the middle cuneate, intracellular and patch-clamp techniques have recently been used in vivo $[4,5,34,36,37]$. It was found that cuneothalamic neurons possess two modes of activity: oscillatory and tonic [5]. During deep anaesthesia, the cuneothalamic neurons showed oscillatory bursting activity. This behaviour changed to the tonic mode when either depolarizing currents were intracellularly injected or the excitatory peripheral receptive field was stimulated. A low-threshold calcium current $\left(I_{T}\right)$ and a hyperpolarization-activated cationic current $\left(I_{h}\right)$ have been postulated to explain the oscillatory bursting activity in vivo [5]. Subsequent in vitro experiments have demonstrated the presence of $I_{h}$ currents in the dorsal column nuclei $[11,44,46]$.

The electrophysiological properties of cuneothalamic neurons are key to understand how the cuneate processes cutaneous information during wakefulness as well as participating in the generation of slow waves during sleep. With these two main objectives in mind, we have resorted to the use of computational models in order to integrate the experimental data in an attempt to answer the following questions. Which ionic currents may explain the activity of the cuneothalamic projection neurons? How do these currents participate in the membrane potential? Which are the firing modes of the middle $\mathrm{CN}$ neurons? What do they mean in the context of the cutaneous transmission through the $\mathrm{CN}$ ?

\section{Methods}

\subsection{Experimental data}

All experiments conformed to Spanish guidelines (BOE 67/1998) and European Communities Council Directive 86/609/EEC, and all efforts were made to minimize the number of animals used. Data were obtained from cats $(2.3-4.5 \mathrm{~kg})$, which were anaesthetized ( $\alpha$-cloralose, $60 \mathrm{mg} \mathrm{kg}^{-1}$, i.p.), paralysed (Pavulon, $1 \mathrm{mg} \mathrm{kg}^{-1} \mathrm{~h}^{-1}$, i.v) and under artificial respiration [5, 34]. Additional doses of anaesthesia were administered when necessary. The dorsal medulla was exposed to insert recording electrodes within the middle main $\mathrm{CN}$ from the level of the obex to $4 \mathrm{~mm}$ caudal to it.

Cuneothalamic cells were identified as antidromically activated by medial lemniscus stimulation according to standard criteria, including the collision test as well as confirmation that the critical interval in the collision was not due to soma refractoriness [5]. To stimulate the PAF fibres a silver ball stimulating electrode was positioned over the ipsilateral dorsal column at $\mathrm{C} 2$.

Intracellular current-clamp records were obtained using the whole-cell technique. A detailed description of the preparation can be found in [5, 34]. 


\subsection{Computational models}

2.2.1. Neuron level. We have followed the single-compartment approach [45] for each cell. The membrane potential for each compartment is computed by using the following expression:

$$
C \frac{\partial V}{\partial t}=-I_{m}-I_{s y n}-I_{\text {inject }}
$$

where $C$ is the membrane capacitance, $I_{m}$ the sum of the ionic currents, $I_{s y n}$ the synaptic current and $I_{\text {inject }}$ the electrical stimulation. We have developed two types of structure.

- Primary afferents. With the aim of providing synaptic stimulation to the cuneothalamic neuron, we have modelled a simple PAF with a fast sodium current and a delayed rectifier potassium current.

- Cuneothalamic cells. There exists experimental evidence (see section 3.1) for the existence of a high-threshold calcium current $I_{L}$, a calcium-activated potassium current $I_{a h p}$, a hyperpolarization-activated cationic current $I_{h}$ and a low-threshold calcium current $I_{T}$. In addition to this, we have introduced a fast sodium current $I_{\mathrm{Na}}$ and a delayed rectifier potassium current $I_{\mathrm{K}}$. The membrane currents will be $I_{m}=I_{\mathrm{Na}}+I_{\mathrm{K}}+I_{L}+I_{a h p}+I_{h}+I_{T}+I_{l}$, where $I_{l}$ represents the contribution of currents associated with passive currents.

2.2.2. Membrane level. The Hodgkin-Huxley (HH) formulation was used as the mathematical model for describing the ionic currents [17-21]. The HH general expression for an ionic current related to a membrane channel is

$$
I_{\text {ion }}=g_{\text {ion }}^{\max } m^{p} h^{q}\left(V-V_{\text {ion }}\right)
$$

where $g_{\text {ion }}^{\max }$ represents the maximum conductance, $V_{\text {ion }}$ the equilibrium potential for such an ion, $m$ the probability of an activation gate being open, $h$ the probability for an inactivation gate being open and $p$ and $q$ the number of activation and inactivation gates of this ionic channel.

Mathematical expressions describing the ionic currents are presented in the appendix.

2.2.3. Molecular level. In order to describe intracellular calcium dynamics we have used Traub's model [54]. The mathematical expression is a differential equation that computes intracellular calcium concentration over time. This rate depends on both calcium current values $\left(I_{L}\right.$ and $\left.I_{T}\right)$ and calcium diffusion processes.

$$
\frac{\mathrm{d}[\mathrm{Ca}]}{\mathrm{d} t}=-K\left(I_{L}+I_{T}\right)-\mu_{d i f}[\mathrm{Ca}]
$$

2.2.4. Synaptic level. We have used Abbott's synaptic model [1]. The expression of the synaptic currents is similar to the HH model:

$$
I_{\text {syn }}(t)=g_{\text {syn }}(t)\left(V-V_{\text {syn }}\right) .
$$

In turn, the conductance $g_{s y n}$ is equivalent to the product $g_{s y n}^{\max } * s$, where $g_{s y n}^{\max }$ is the maximum conductance, and $s$ the probability of the postsynaptic currents being open. This probability is calculated by a differential equation that depends on the quantity of released neurotransmitter $T\left(V_{\text {pre }}\right)$. This variable is, in turn, a function of the difference between the presynaptic membrane potential $\left(V_{\text {pre }}\right)$ and a threshold value $(v t)$ :

$$
T\left(V_{\text {pre }}\right)=\frac{a}{b+d \exp \left[-\left(V_{\text {pre }}-v t\right) / v_{\text {syn }}\right]} \text {. }
$$

To analyse the relationship between firing modes and synaptic activity we have introduced the alpha function, which represents the conductance associated with the synaptic current. 
Table 1. Ionic current parameters for cuneothalamic projection neurons: maximum conductances and equilibrium potentials.

\begin{tabular}{lccccccc}
\hline & $I_{\mathrm{Na}}$ & $I_{\mathrm{K}}$ & $I_{L}$ & $I_{T}$ & $I_{\text {ahp }}$ & $I_{h}$ & $I_{l}$ \\
\hline$g_{\text {ion }}^{\max }(\mathrm{nS})$ & 10 & 2.5 & 0.01 & 0.1 & 1.2 & 0.05 & 0.001 \\
$V_{\text {ion }}(\mathrm{mV})$ & 40 & -70 & 100 & 100 & -70 & -43 & -62 \\
\hline
\end{tabular}

Table 2. General parameters for cuneothalamic projection neurons.

\begin{tabular}{llllllllll}
\hline $\begin{array}{l}C \\
(\mathrm{nF})\end{array}$ & $\begin{array}{l}R \\
(\mathrm{M} \Omega)\end{array}$ & $\begin{array}{l}K \\
\left(\mathrm{nA}^{-1} \mathrm{mseg}^{-1}\right)\end{array}$ & $\mu_{\text {dif }}$ & $\begin{array}{l}g_{S}^{\max } \\
(\mathrm{nS})\end{array}$ & $a$ & $b$ & $d$ & $\begin{array}{l}v_{t} \\
(\mathrm{mV})\end{array}$ & $v_{s y n}$ \\
\hline 0.07 & 50 & 0.05 & 2 & 3 & 1 & 1 & 1 & -30 & 2 \\
\hline
\end{tabular}

This function determines the shape of the postsynaptic potential generated after appropriate stimulation [26]. The mathematical expression is

$$
g_{s y n}(t)=g_{\sin }^{\max } \frac{t-t^{\prime}}{\tau} \exp \left(-\frac{t-t^{\prime}-\tau}{\tau}\right)
$$

where $g_{\sin }^{\max }$ is a constant, $t^{\prime}$ the initial time and $\tau$ the time constant. The synaptic conductance value will be zero if the presynaptic potential is less than a certain threshold and it will be equal to the alpha function above such a threshold.

2.2.5. Model parameters. Maximum conductances and equilibrium potentials for each ionic current are shown in table 1. Conductance values have been adjusted to reproduce current injection experiments (see section 3.1). These values have been tested by comparison of simulations and experiments under three different situations (see section 3.2): spontaneous activity, sustained electrical stimulation and synaptic stimulation. In table 2 values related to the passive properties of cuneothalamic neurons, intracellular calcium dynamics for the Traub model and synaptic currents following the Abbott model are also shown.

\subsection{Computational tools}

We have chosen two popular neurosimulators: $X P P$ and neuron. The XPP (Xwindows PhasePlane), developed by Ermentrout [12], was used to test the ionic currents and to produce most of the results shown in this paper. Due to the stiffness of the model, the Gear adaptive numerical method [14] was finally selected. With this integration scheme we had to reach a tolerance value that guarantees stability as well as avoiding large-scale deviations between numerical and algebraic solutions.

Neuron, developed by Hines and Moore, offers tools for implementing realistic models of neurons and circuits [16]. By default, the simulator uses the Hines integration method, an optimized algorithm that combines the benefits of both Crank-Nicholson and Euler methods.

$X P P$ simulations were carried out on a Sun Sparcstation 20 workstation and neuron simulations on a PC with a $600 \mathrm{MHz}$ AMD K7 processor.

\section{Results}

This section is organized in three subsections: ionic currents, validation and prediction. In the first one we present the current-injection experiments [5] that: 


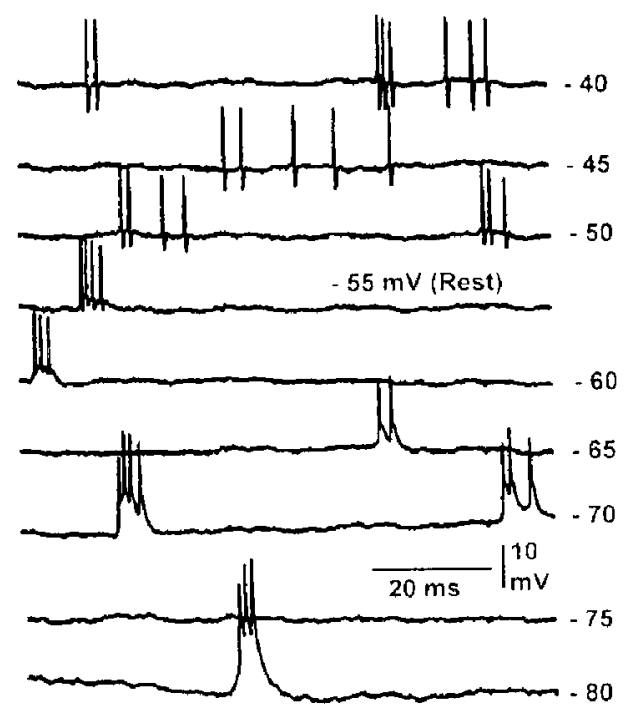

Figure 1. Response of cuneothalamic projection cells to membrane polarization. Hyperpolarization from rest $(-55 \mathrm{mV})$ induces bursting activity with two to four spikes per burst. Depolarization from rest generates tonic activity with a fast post-spike hyperpolarization.

(1) provide evidence for the ionic currents and

(2) were used to tune the model with the experimental data.

Afterwards the model is validated with neuronal activity recorded in vivo, in anaesthetized cats, under three different experimental protocols [4, 5, 33, 38, 39]. Finally, computer simulations are shown that predict both neuronal behaviour in the case of transition from sleep to wakefulness, and firing modes in simulated wake conditions under excitatory/inhibitory synaptic stimulation.

\subsection{Ionic currents}

Intracellular current injection in the cuneothalamic projection neuron demonstrates different neuronal activity between hyperpolarized and depolarized membrane levels. Figure 1 shows that hyperpolarization from rest $(-55 \mathrm{mV}$ for this cell) induces slow potentials that generate bursts of conventional spikes. On the other hand, depolarization from rest induces single-spike activity with a post-spike hyperpolarization. These findings suggest that distinct underlying ionic currents are activated at different membrane potentials.

Figure 2(A) illustrates in detail what happens when hyperpolarization current pulses of $300 \mathrm{~ms}$ duration are applied. The pulse uncovers a depolarizing sag, which increases with current amplitude $(-0.5 \mathrm{nA})$. This phenomenon points to a hyperpolarization-activated cationic current, $I_{h}$, which depolarizes the neuron after a certain degree of hyperpolarization is achieved. At the end of the greater hyperpolarization pulse, the neuron generates a slow rebound potential that induces a burst of two sodium spikes. This excitatory rebound is presumably due to a low-threshold calcium current, $I_{T}$. Figure 2(B) illustrates the behaviour of the cuneothalamic neuron model. Dynamics of both $I_{h}$ and $I_{T}$ currents are shown in figures $2(\mathrm{C})$ and (D). At the beginning of the hyperpolarizing pulse $I_{h}$ is activated, thus impeding a deeper hyperpolarization, and $I_{T}$ is also de-inactivated. When the pulse ends, the $I_{T}$ currents are activated, which raises the membrane potential until the $I_{\mathrm{Na}}$ threshold is reached. Thereafter a burst of spikes appears in the same manner as it is observed in real neurons. 

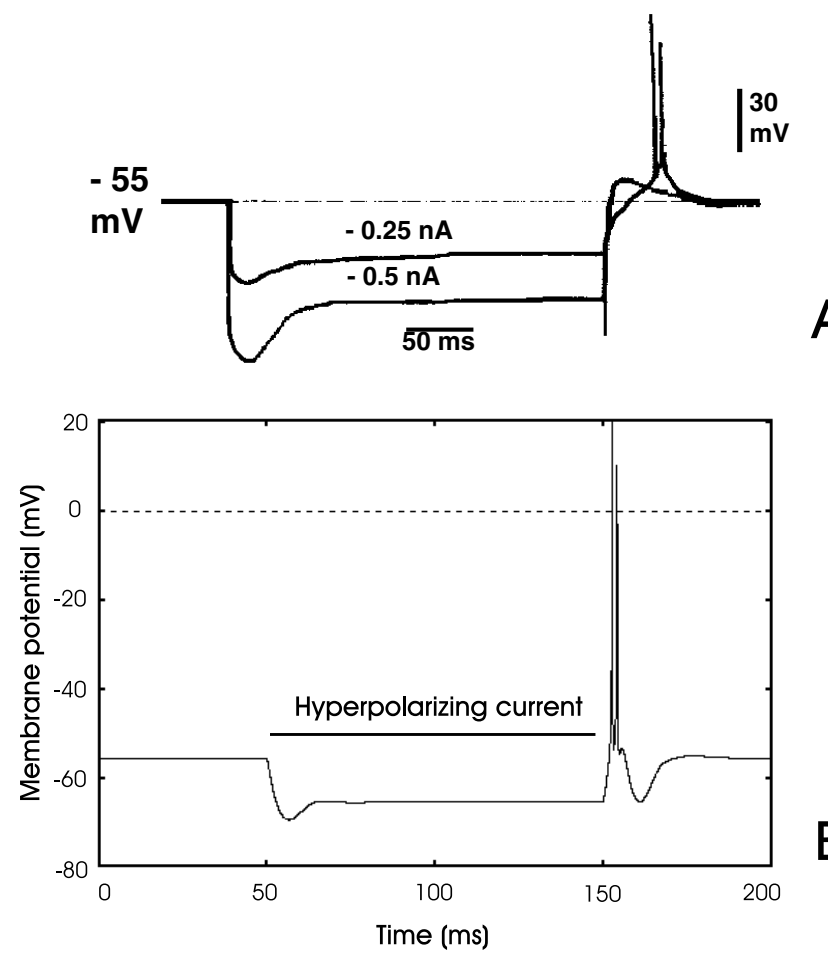

B
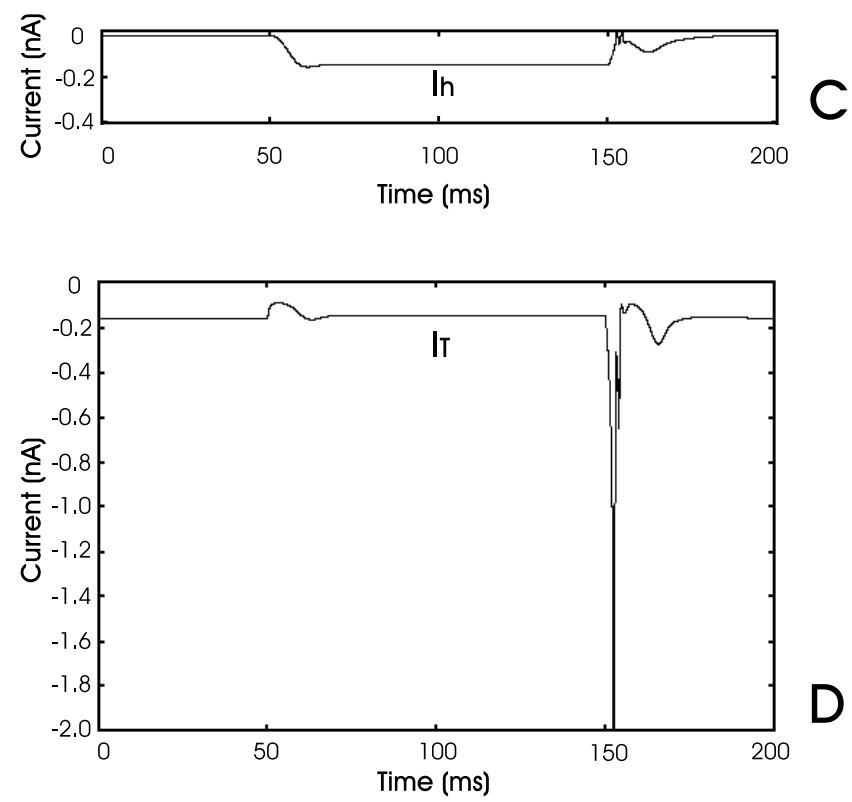

Figure 2. Experimental (A) and simulated response (B)-(D) to hyperpolarizing current pulses. The cell shows a depolarizing sag (A), also reproduced in the simulations (B), which is presumably generated by a $I_{h}$ current $(\mathrm{C})$, that increases in amplitude with the current pulse. When the pulse ceases, a rebound potential appears, probably induced by the activation of a $I_{T}$ current (D) (previously de-inactivated at the beginning of the pulse), that induces bursting activity. 
Table 3. Description of ionic currents used in the projection neuron simulations.

\begin{tabular}{|c|c|c|c|c|}
\hline & Description & Activated by & Function & References \\
\hline$I_{\mathrm{Na}}$ & $\begin{array}{l}\text { Fast and transient } \\
\text { sodium current }\end{array}$ & Depolarization & $\begin{array}{l}\text { Generates action } \\
\text { potentials }\end{array}$ & {$[22]$} \\
\hline$I_{\mathrm{K}}$ & $\begin{array}{l}\text { Delayed rectifier } \\
\text { potassium current }\end{array}$ & Depolarization & Repolarizes & {$[41]$} \\
\hline$I_{L}$ & $\begin{array}{l}\text { High-threshold } \\
\text { calcium current }\end{array}$ & Depolarization & Introduces calcium & [29] \\
\hline$I_{T}$ & $\begin{array}{l}\text { Low-threshold } \\
\text { calcium-current }\end{array}$ & Depolarization & Introduces calcium & $\begin{array}{l}{[9]} \\
{[55]}\end{array}$ \\
\hline$I_{a h p}$ & $\begin{array}{l}\text { Potassium } \\
\text { current }\end{array}$ & $\begin{array}{l}\text { Intracellular } \\
\text { calcium }\end{array}$ & $\begin{array}{l}\text { Repolarizes when } \\
\text { calcium is high }\end{array}$ & {$[56]$} \\
\hline$I_{h}$ & $\begin{array}{l}\text { Cation } \\
\text { current }\end{array}$ & Hyperpolarization & Controls frequency & {$[42,49]$} \\
\hline
\end{tabular}

Post-spike hyperpolarizations during spontaneous activity are another interesting feature of cuneothalamic neurons (figure 3). This behaviour can be explained by the activation of a special type of potassium current that would repolarize the cell. As shown in figure 3(A), the magnitude of that hyperpolarization increases with bursting. During bursting, a calciumdependent mechanism, consisting of a high-threshold calcium current, $I_{L}$, and a calciumdependent potassium current, $I_{a h p}$, is probably involved. $I_{L}$ would introduce calcium into the cell during bursting and $I_{a h p}$ would control the post-spike hyperpolarization. Figure 3(B) shows the behaviour of our model in which the ionic current parameters were adjusted to reproduce the observed activity. Ionic current activity is shown in figure 3(C). When two spikes per burst appear, $I_{a h p}$ shows a peak of activity which explains the increased hyperpolarization.

In conclusion, current injection experiments provide evidence for the existence of a set of ionic currents responsible for the behaviour illustrated in figure 1. These currents, whose features are summarized in table 3, were used in the construction of the computational model of the cuneothalamic neurons.

\subsection{Validation}

3.2.1. Spontaneous activity Figure 4(A) shows the spontaneous activity recorded from a slow adapting cuneothalamic neuron. The oscillatory bursting activity had the following salient features:

(1) the action potentials were grouped together with an intra-burst frequency of up to $1000 \mathrm{~Hz}$,

(2) each burst consisted of two to five spikes,

(3) the resting membrane potential varied between -60 and $-65 \mathrm{mV}$,

(4) the action potentials reached peaks of $0-20 \mathrm{mV}$, and

(5) the inter-burst interval varied between 60 and $2 \mathrm{~Hz}$.

The spontaneous activity of the model is shown in figure 4(B). The membrane potential has an oscillatory pattern with three to four action potentials per burst. The baseline is situated at $-63 \mathrm{mV}$, the inter-burst interval is $20 \mathrm{~ms}$ and the peak potentials vary between 8 and $11 \mathrm{mV}$, with all these values being in the same range as the experimental ones.

The dynamics of the salient ionic currents are shown in figures 4(C) and (D). The lowthreshold calcium current induces bursting activity by activating the $I_{\mathrm{Na}}$ current. Each action potential activates $I_{L}$, thus introducing calcium inside the cell. On each burst, the amount of intracellular calcium increases as more spikes are generated. This trend finalizes when 


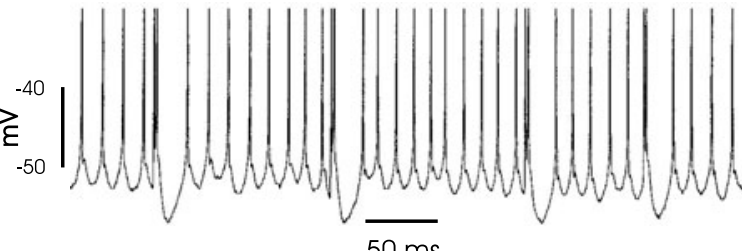

A

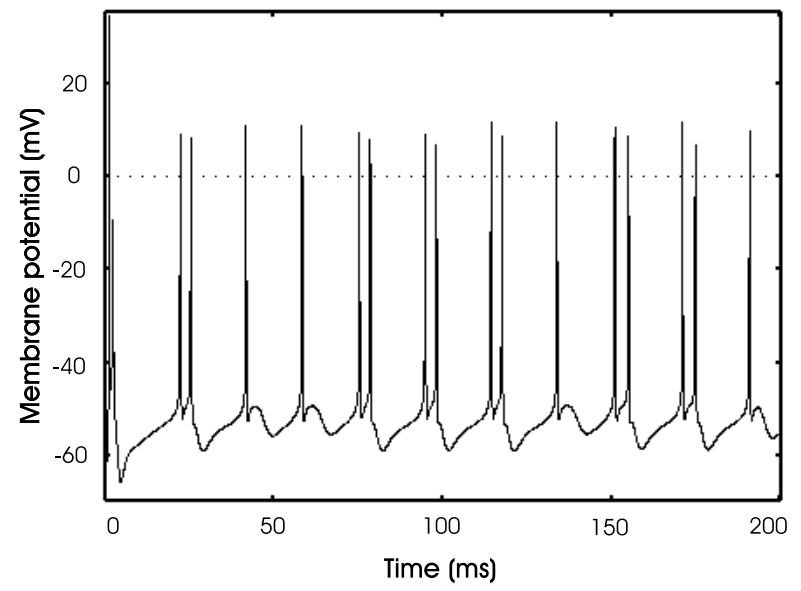

B

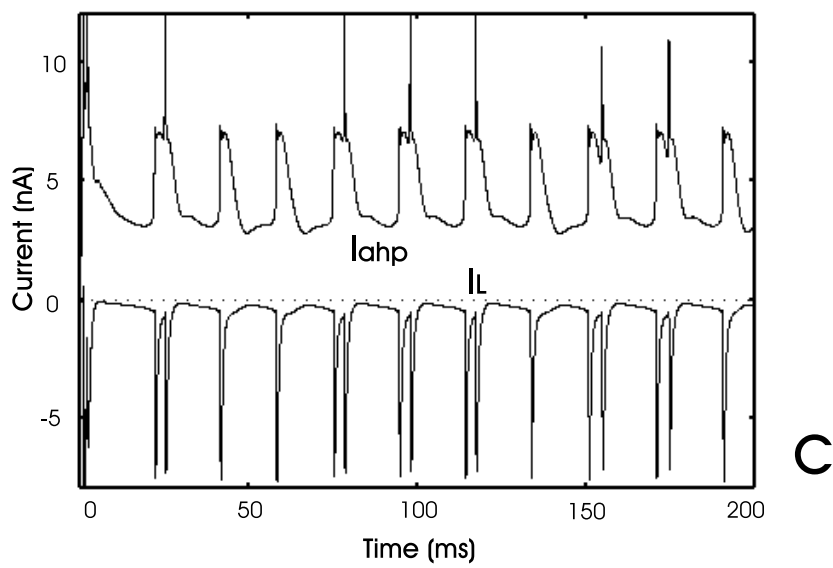

Figure 3. Post-spike hyperpolarization depends on the number of spikes per burst. The neuron illustrated in (A) shows a post-spike hyperpolarization that increases with bursting. In (B) computer simulations reproduce this firing pattern. A calcium-dependent potassium current, $I_{a h p}$, and a lowthreshold calcium current, $I_{L}$, are proposed to explain this activity (C).

the amount of intracellular calcium reaches a value that is sufficiently high to activate $I_{a h p}$. This current, in cooperation with $I_{\mathrm{K}}$ (delayed rectifier), is responsible for repolarizing and hyperpolarizing the membrane potential, thus terminating the burst. The hyperpolarization activates $I_{h}$ and de-inactivates $I_{T}$, thus leading, at the end of the hyperpolarization, to the activation of $I_{T}$. After that, the activity pattern continues on and a new burst begins. The elimination of calcium is mediated by diffusion processes, like sodium-calcium pumps, that expel it to the extracellular medium. 

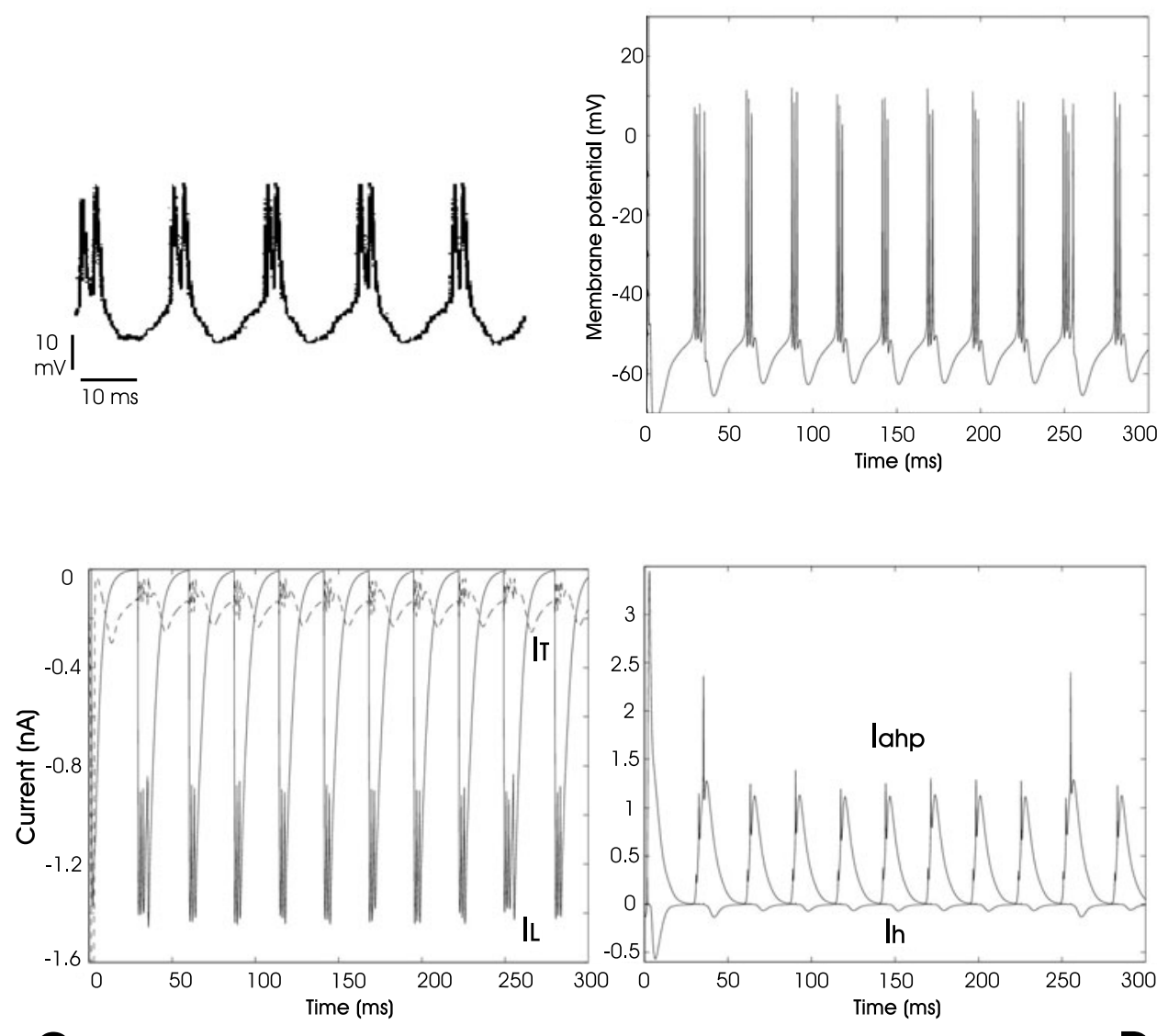

C

Figure 4. Experimental and simulated behaviour of spontaneous neuronal activity. Intracellular recordings obtained in vivo show a bursting oscillatory mode observed during spontaneous activity (A). Simulated membrane potential (B). High-threshold $\left(I_{L}\right)$ and low-threshold $\left(I_{T}\right)$ calcium currents $(\mathrm{C})$. Calcium-dependent potassium current $\left(I_{a h p}\right)$ and hyperpolarization-activated cationic current $\left(I_{h}\right)(\mathrm{D})$.

3.2.2. Response to current pulses. Under intracellular injection of positive current pulses of $0.2 \mathrm{nA}$ the cuneothalamic neuron changes to tonic activity (figure 5(A)). It is shown that:

(1) as the cell is depolarized, the oscillatory bursting behaviour is replaced by tonic firing during the stimulation period,

(2) the membrane potential rises between 5 and $10 \mathrm{mV}$,

(3) the action potential peak is similar to what was seen during the bursting mode, and

(4) a hyperpolarization appears after the elimination of the current pulse.

In the tonic mode, not all currents participate actively in the generation of the membrane potential. Either injection of positive current pulses (figure 5(A)) or receptive field stimulation (see section 3.2.3) establish a sufficiently depolarized membrane potential to impede the significant activation of $I_{h}$ as well as of $I_{T}$. These currents require hyperpolarization, to activate 

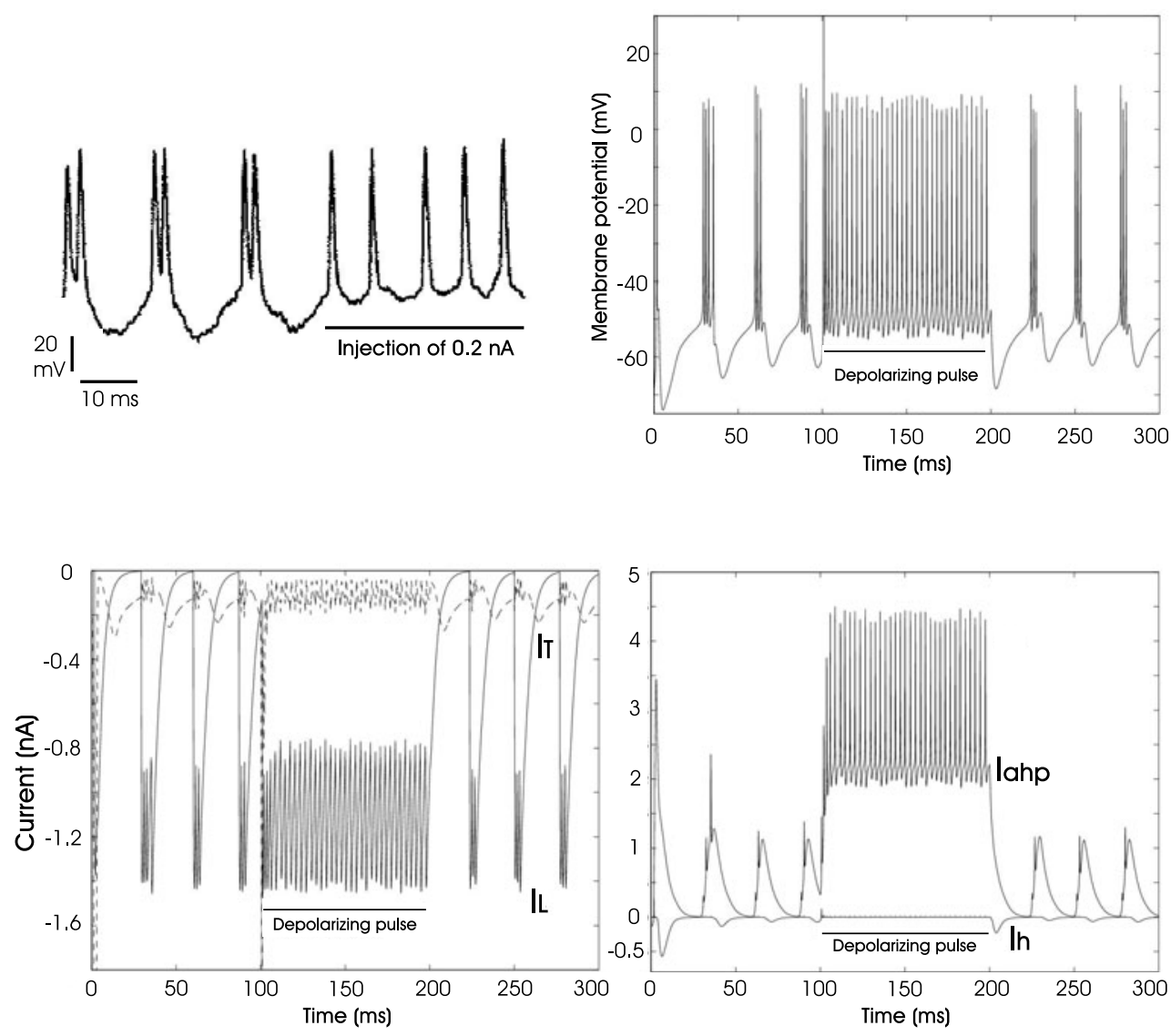

C

Figure 5. Experimental and simulated responses to depolarizing current pulses. Intracellular recordings show a single-spike tonic mode (A). Simulated membrane potential (B). High-threshold $\left(I_{L}\right)$ and low-threshold $\left(I_{T}\right)$ calcium currents $(C)$. Calcium-dependent potassium current $\left(I_{a h p}\right)$ and hyperpolarization-activated cationic current $\left(I_{h}\right)(\mathrm{D})$.

$I_{h}$ and de-inactivate $I_{T}$, as well as a subsequent depolarization from sufficiently negative levels of potential, to activate $I_{T}$, neither of which is ever attained under membrane depolarization.

Figure 5(B) shows the membrane potential for the computational model. It rises $8 \mathrm{mV}$ in response to $1 \mathrm{nA}$ current injection. The spike generation rate is similar to that of the bursting mode, and is determined by the activity of $I_{\mathrm{Na}}, I_{\mathrm{K}}$ and $I_{L} . I_{L}$ shows a similar behaviour as during the bursting mode, but $I_{T}$ diminishes notably (figure 5(C)). $I_{a h p}$ is incremented with respect to the bursting mode (figure 5(D)), due to a greater calcium input. The accumulation of calcium establishes high values of $I_{a h p}$, but it does not succeed in forcing hyperpolarization as the current pulse impedes it. As a result the neuron continues responding to the current pulse in a practically indefinite manner. The tonic mode only comes to an end when the current pulse is removed, and $I_{a h p}$, which has been forced to increment its activity, hyperpolarizes the membrane more than is usually seen during the bursting mode. 

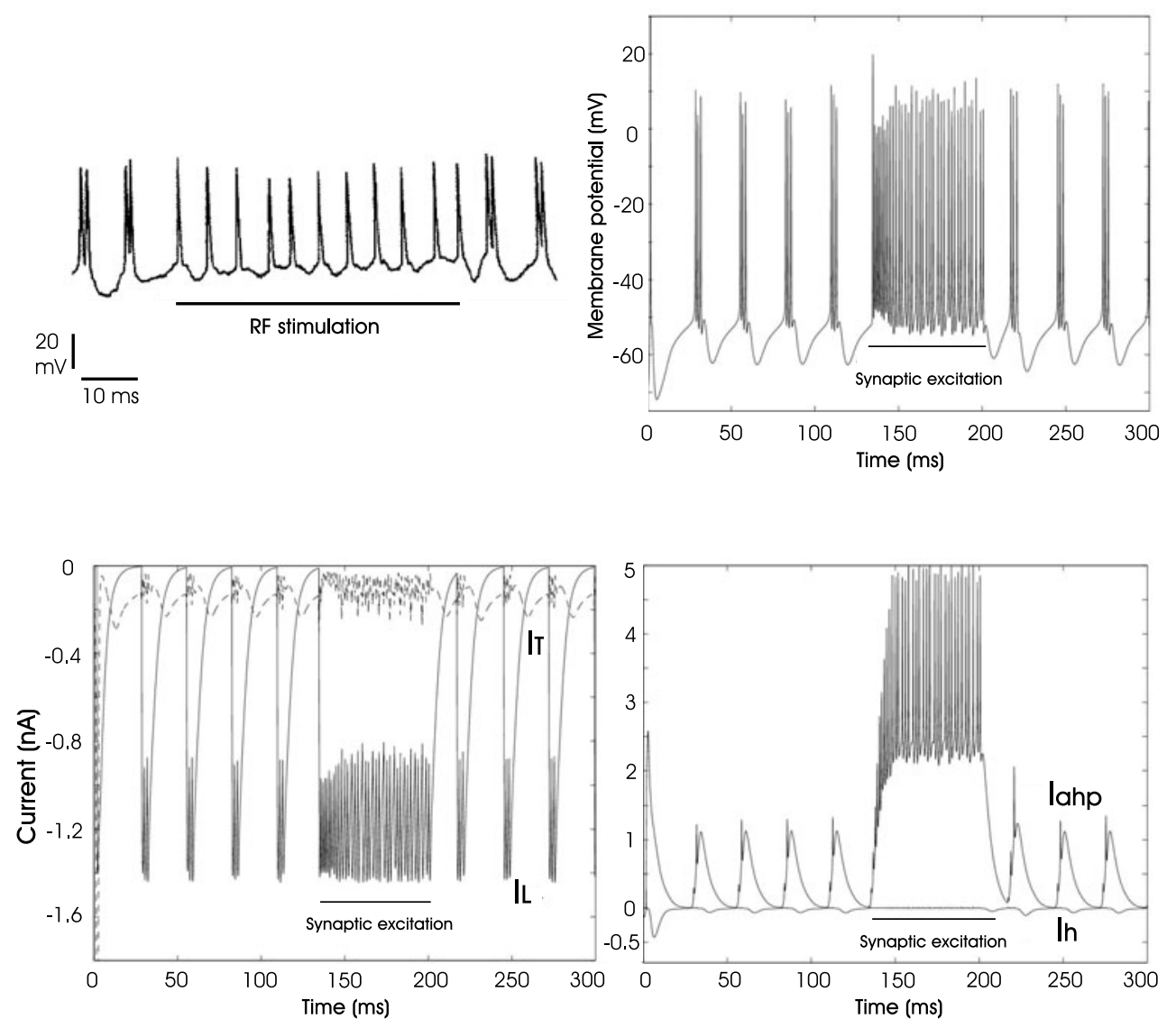

C

Figure 6. Experimental and simulated responses to synaptic excitation. Single-spike tonic mode observed to $70 \mathrm{~ms}$ synaptic excitation (A). Simulated membrane potential (B). High-threshold $\left(I_{L}\right)$ and low-threshold $\left(I_{T}\right)$ calcium currents $(\mathrm{C})$. Calcium-dependent potassium current $\left(I_{a h p}\right)$ and hyperpolarization-activated cationic current $\left(I_{h}\right)(\mathrm{D})$.

3.2.3. Response to synaptic input. Synaptic stimulation in vivo shows very similar behaviour (figure 6(A)) to that shown with current pulses. In order to generate synaptic input at a computational simulation level, we have implemented a simple afferent fibre with sodium and potassium currents. Figure 6(B) shows the model behaviour in response to an input at a frequency of $200 \mathrm{~Hz}$. The appearance of the tonic mode is very similar to the one seen in the previous section. However, some details with regard to $I_{L}$ and $I_{a h p}$ should be pointed out. In the tonic mode the former has a greater decrease between spikes than with current pulses. The reason for this lies in the fact that we are simulating synaptic stimulation by means of a single synapse, and therefore there are silent intervals between postsynaptic potentials in which the neuron is not being stimulated.

Regarding the calcium-dependent potassium current, a more staggered increase of $I_{a h p}$ is observed because the inflow of calcium during synaptic stimulation is less sharp than during current injection 


\subsection{Prediction}

The cuneothalamic model was matched in section 3.2 against experimental data recorded in anaesthetized cats. Under these conditions, the observed oscillatory bursting behaviour is the basic firing pattern associated with the sleep state. Now we question the model for wakefulness: which mechanisms can drive the transition from sleep to wakefulness? Which firing modes, in wake conditions, could be observed under different synaptic input?

3.3.1. Transition from sleep to wakefulness. In the thalamus, the activation of cholinergic and noradrenergic neurons of the brainstem produces a prolonged depolarization of thalamocortical cells [52]. The underlying mechanisms involve, among other things, the activation of muscarinic receptors by acetylcholine, and $\alpha_{1}$-adrenoreceptors by noradrenaline, which reduce the activity of $\mathrm{K}^{+}$conductance. In the $\mathrm{CN}$, afferents from the brain stem [50] and/or from the cerebral cortex [39] could induce similar effects and be in charge of inducing the transition from sleep to wakefulness. Therefore, we have simulated the effect of those afferents by blocking, in our model, the $I_{a h p}$ current. Figure 7(A1) shows that the number of action potentials increased significantly and the membrane did not hyperpolarize. The bursting discharge is again obtained as soon as the $I_{a h p}$ current is unblocked. The $I_{L}$ current, which has no inactivation gates, not only remains activated during the $I_{a h p}$ current blockade, but also increases (figure 7(A2)). Sleep-wakefulness transition could also be triggered by prolonged depolarization driven either by a sustained cutaneous stimulation or by cortical excitatory descendant fibres. The depolarization could set a membrane potential in which significant activation of $I_{h}$ as well as of $I_{T}$ is impeded. We have simulated the prolonged depolarization during sleep (bursting oscillatory) (figure 7(B)). As a result the membrane potential baseline rises significantly and a single-spike tonic behaviour appears. Therefore it seems that the cuneothalamic cell is ready to reach the wake state through different driven mechanisms: some based on a direct effect on ionic channels and others based on a global effect involving a change in the membrane potential.

3.3.2. Firing modes and synaptic activity. The dependence between firing modes and synaptic activity is a main issue in the study of cuneothalamic neurons in order to predict their behaviour when embedded in the cuneate circuitry. For this study we have implemented our model in neuron and used the alpha function to manipulate three meaningful parameters: the time constant, $\tau$, the magnitude of the postsynaptic potential, $g_{\sin }^{\max }$, and the synaptic reversal potential, $V_{\text {syn }}$.

First we have focused on excitatory synapses $\left(V_{\text {syn }}=0 \mathrm{mV}\right)$. If the magnitude of the synapse is sufficiently high, $g_{\sin }^{\max }=0.03 \mathrm{mS}$, and the time constant has a moderate value, $\tau=4 \mathrm{~ms}$, the model generates a train of single spikes (figure 8(A)). This configuration could correspond, for example, to connections established by afferent inputs. These synapses have been reported to be robust enough to ensure the transmission of cutaneous information [2]. On the other hand, if the magnitude of the synapse is decreased, $g_{\sin }^{\max }=0.005 \mathrm{mS}$, and the time constant is sufficiently high, $\tau=16 \mathrm{~ms}$, the synaptic stimulation would generate a membrane depolarization without spikes (figure $8(B)$ ). This kind of stimulation would increase the probability of firing for further incoming stimuli. In the discussion we will deal with the possible functional role of this neuronal response.

The inhibitory synapses were modelled with $V_{\text {syn }}$ in the range between -60 and $-90 \mathrm{mV}$. The neuron response is totally different depending on the value of $V_{\text {syn }}$. For weak inhibition, $V_{\text {syn }}$ between -60 and $-75 \mathrm{mV}$, the membrane is hyperpolarized and a silent response is observed (figure $9(\mathrm{~A})$ ). For deeper inhibition, $V_{\text {syn }}$ between -75 and $-90 \mathrm{mV}$, the membrane is 


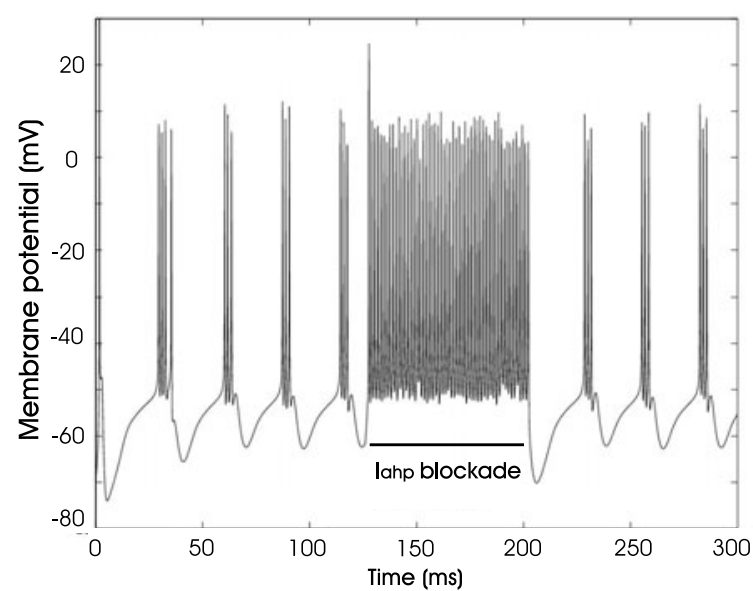

$\mathrm{Al}$

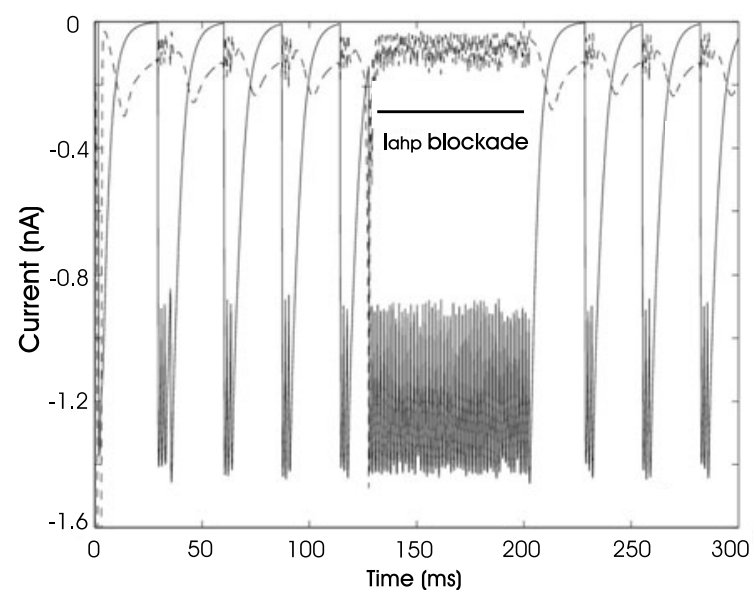

$\mathrm{A} 2$

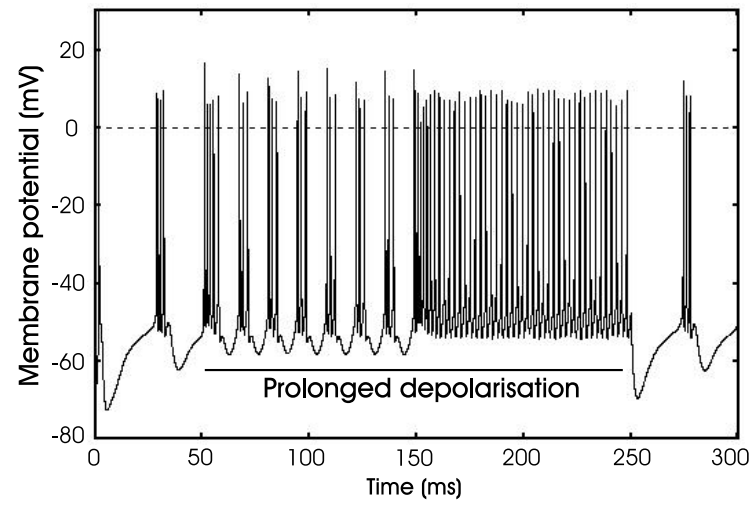

Figure 7. Transition mechanisms from sleep to wakefulness. The $I_{a h p}$ current finalizes the bursting activity. After $I_{a h p}$ blockade the generation of action potentials increases significantly (A1). $I_{T}$ decreases notably during $I_{a h p}$ blockade due to the absence of membrane hyperpolarization (A2). A prolonged depolarization driven by cutaneous and/or cortical afferents also abolishes the oscillatory bursting activity. 

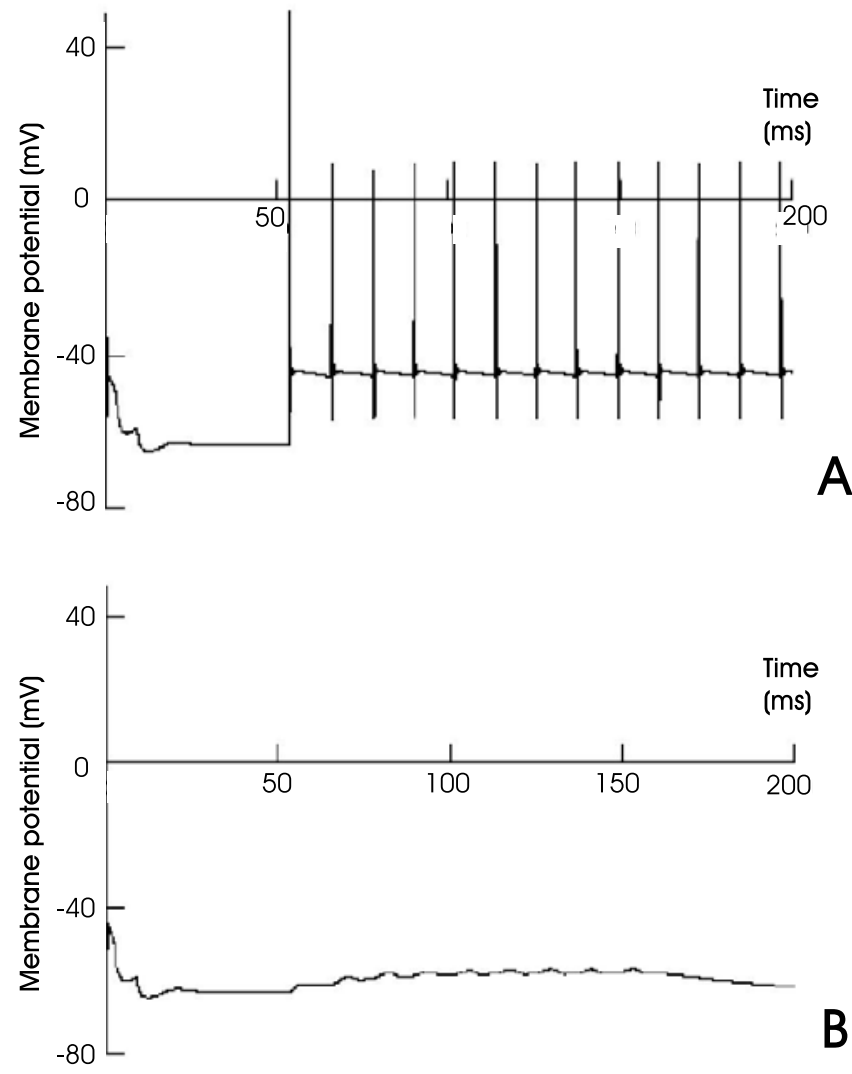

Figure 8. Effect of synaptic excitation. Excitatory synapses with high synaptic magnitudes and moderate time constants induce a train of single spikes (A). Low synaptic magnitudes and high time constants depolarize the membrane without generating spikes (B).

also hyperpolarized but there appears a burst of spikes at the end of the stimulation (figure 9(B)). This result can be explained by considering the activation of both $I_{h}$ and $I_{T}$. The generation of two different responses depending on the synaptic reversal potential points to the existence of two types of inhibitory mechanism in the CN. We have forced our model to show both responses with only one synaptic type and we got either a silent or a burst response. Again, we will deal in the discussion section with the meaning of these results.

\section{Discussion}

\subsection{Firing modes and physiological mechanisms in the cuneate}

Firing modes depend primarily on the general brain state, synaptic activity and intrinsic properties. There are two well established brain states, sleep and wakefulness, which determine the activity pattern of the overall brain and have a decisive impact on neuronal behaviour [40, 52]. In each state, synaptic activity defines the inputs at the level of single neurons. Inputs can be categorized as (1) afferent/sensory signals and (2) modulatory signals. The later set up the membrane level and therefore determine the ionic currents to be activated by afferent/sensory inputs. In what follows we discuss the dependence between firing modes, general brain states and synaptic activity in the $\mathrm{CN}$. 

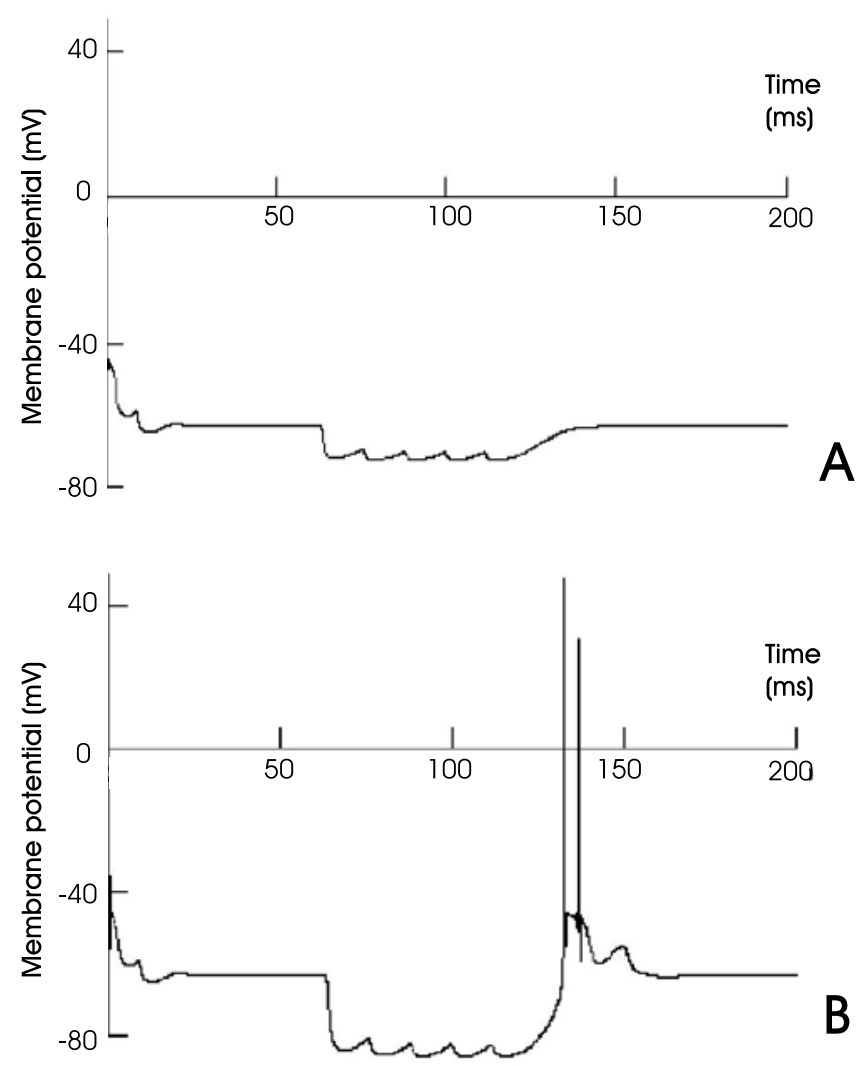

Figure 9. Effect of synaptic inhibition. Inhibitory synapses with $V_{\text {syn }}=-70 \mathrm{mV}$ do not elicit rebound-propagated responses in the cuneothalamic cell (A). Setting $V_{\text {syn }}$ to $-90 \mathrm{mV}$ induces a greater hyperpolarization, activating low-threshold currents that induce bursting-rebound discharges.

4.1.1. Sleep state. The experimental data reported here were recorded in anaesthetized cats, thus mimicking sleep conditions. Sleep is mainly characterized by the synchronization of large populations of neurons, which force an oscillatory behaviour at the single-neuronal level. In the cuneate, the oscillations present a bursting pattern (see figure 4(A)) that is generated if the following conditions are fulfilled: (1) a sufficient hyperpolarized membrane level and (2) an appropriate set of ionic currents $\left(I_{h}, I_{T}, I_{L}\right.$ and $\left.I_{a h p}\right)$. The first point is supported by experimental evidence (see figure 1) and was needed when tuning the computational model. The second point was indicated by intracellular experiments and found to be required in the model in order to reproduce the experimental results (see figures 2 and 3). In this scenario, synaptic activity seems to play a fundamental role to modulate the frequency of the bursting pattern and produce slow waves, typically found during deep sleep.

4.1.2. Wake state. Once the model had been tuned with data recorded during anaesthesia, we used it to predict behaviour during wakefulness. First, we simulated the transition from sleep to wake state by raising the membrane potential to a level in which only high-threshold currents could be activated. There could be two main triggering signals: internal and external. The former could be generated from the brain stem modulating the neuronal cuneothalamic 
ionic current dynamics, such as the $I_{a h p}$ current. The later could involve cutaneous and/or cortical input to provide prolonged excitation to trigger the transition.

The results in section 3.3 show the relationship between firing modes in the wake state and synaptic configuration. Now we will discuss the relationship between these synaptic configurations and the cuneate network.

- Afferent excitation. Cutaneous stimulation induces synaptic excitation on cuneothalamic cells [6]. These synapses are robust enough [2] to ensure the generation of action potentials and the transmission of incoming signals. When these synaptic conditions were introduced in the model (high synaptic magnitudes and moderate time constants), spike trains were generated. The model predicts that there can be a 1:1 ratio between input-output spikes. This ratio is maintained up to $500 \mathrm{~Hz}$ of stimulating frequency (not shown).

- Afferent inhibition. Canedo and Aguilar [6] have reported experimental evidence for the existence of centre-surround receptive fields in the $\mathrm{CN}$. The surround inhibition could be in charge of impeding the generation of postsynaptic potentials to avoid the transmission of information if afferent excitation is not strong enough. If the inhibitory synapse is modelled with $V_{\text {syn }}=-75 \mathrm{mV}$, weak inhibition, a silent response after stimulation is observed in the model. On the other hand, surround inhibition could play a role in detecting dynamic stimuli. This would mean that the stimulus could induce inhibition-excitation sequences during its motion. Deep inhibitions, with $V_{s y n}=-90 \mathrm{mV}$, generate bursting (see figure 9) that could signal the detection of dynamic stimuli. This interpretation would reinforce a recent trend that considers bursting as an important codification pattern [31].

- Recurrent excitation/inhibition. Lemniscal stimulation has revealed both excitatory and inhibitory recurrent effects [7]. Excitatory connections, for instance, can be in charge of depolarizing the cell, thus facilitating neuronal responses. This mechanism has been postulated to help the synchronization of neighbouring cells [7], and can be explained by synapses with moderate synaptic strength on membranes and high time constants (see figure 8). Inhibitory connections, on the other hand, could block the response of other cuneothalamic cells and therefore might be associated with weak inhibitory input $\left(V_{\text {syn }}=-75 \mathrm{mV}\right)$.

- Cortical inhibition. Corticospinal collaterals and corticocuneate fibres also make synaptic contact with cuneate neurons [4]. One of their possible effects is to inhibit cuneothalamic neurons. There are several possible interpretations for this inhibition: (1) tonic inhibition, (2) attention signal and (3) prediction signal. The first one could be related to weak inhibition, $V_{\text {syn }}=-75 \mathrm{mV}$, and be in charge of suppressing signals not relevant to the cortex at any given time. The second one could be a signal generated by some attentional mechanism that would prepare the cell to fire by recruiting low-threshold currents. This could be done only by deep hyperpolarizations which are induced by strong inhibitory synapses $\left(V_{\text {syn }}=-90 \mathrm{mV}\right)$. The last one could work as a signal that would predict the input from the outside world. If the cell does not fire, this would mean that the prediction was right. If not, the cell would fire (excitation greater than inhibition) or a burst would be generated (inhibition greater than excitation). The occurrence or absence of firing would thus determine a mechanism to improve the predictive power of the system.

- Cortical excitation. Gordon and Jukes [15] showed that the sensorimotor cortex sends excitatory descendant fibres to the medial zone of the dorsal column nuclei. These connections may facilitate the cutaneous information flow through the cuneate, a phenomenon recently reported by Canedo and Aguilar [6]. This could be done if the synaptic stimulation depolarizes the cell without generating spikes which would be possible by having afferent inputs with low synaptic strength $g_{\sin }^{\max }$ and high time constant $\tau$. 


\subsection{Comparison with other models}

Direct comparison with other models of cuneothalamic neurons is not possible because, as far as we know, this is the first attempt to model the CN. However, we can discuss similarities and differences with two computational models proposed for the somatosensory thalamus. The first of these models was developed by McCormick and Huguenard [41]. Their work is based on solid knowledge about the underlying ionic currents, which were studied in both in vitro and in vivo preparations [22-25, 27, 28, 40, 42, 43, 51, 55]. The model was mainly used to study the participation of each ionic current in bursting activity. The second model was proposed by Lytton et al [32]. There are no significant differences between both models and again their goal is to analyse the oscillatory bursting activity and how synaptic activity can modulate its frequency.

The similarities between our and their models are (1) the core of ionic currents and (2) the tonic and oscillatory activity patterns. Some differences, however, exist:

(1) a number of ionic currents considered in the thalamocortical models (a persistent sodium current $I_{\mathrm{Na} p}$, a potassium current $I_{k 2}$ and a fast potassium current $I_{A}$ ) do not seem to play a role in cuneothalamic neurons and

(2) the firing modes in wakefulness.

There are several ways to explain the greater complexity, in terms of ionic currents, observed in thalamic neurons:

(1) the thalamus is a newer and more complex structure than the cuneate and/or

(2) in vitro experiments used in the study of thalamic ionic currents can reveal physiological properties that could not have a precise functional meaning in intact-brain preparations.

But the main difference is our perspective. Our goal is to simulate wake conditions in the cuneothalamic model and predict firing modes depending on different configurations of synaptic activity. Intact-brain preparations should be used to test our predictions [53].

\subsection{Conclusions}

In conclusion, we emphasize the following.

The first computational models of the cuneate nucleus reproduce experimentally observed tonic and oscillatory modes. In this work, the simulation results have been compared with those observed experimentally under three different protocols (spontaneous activity, response to intracellular injection of current pulses and response to synaptic stimulation). The oscillatory and tonic modes shown by cuneate neurons can be explained on the basis of a single set of ionic currents. These types of response have been observed in vivo [5], as well as in vitro [44].

The $I_{h}$ and $I_{T}$ currents define the inter-burst period during the oscillatory mode in projection neurons. The activation of $I_{h}$ depolarizes the neuron and shortens the period of time that is necessary to generate a new burst. $I_{T}$, on the other hand, is essential for completing the depolarization and for activating the sodium current, $I_{\mathrm{Na}}$. Recent data obtained in vitro [44], from a brain stem-spinal cord preparation [11], and in vivo [5], have demonstrated the existence of the $I_{h}$ current in cuneothalamic cells. 
The $I_{L}$ and $I_{a h p}$ currents determine the number of spikes per burst in the oscillatory mode. The $I_{L}$ current plays a dual role: it maintains the continuous generation of spikes during each burst and introduces calcium into the cell. Conversely, $I_{a h p}$ determines the duration of each burst.

Firing modes could encode different physiological mechanisms. Different firing modes are generated under a variety of synaptic stimulation conditions. Spike trains and depolarized silent behaviour can be driven by excitatory synapses. These synapses could be associated with afferent excitatory input and cortical modulation respectively. Silent hyperpolarizations and burst responses are generated through inhibitory synapses that could mediate afferent inhibition and cortical filtering. Finally, bursting oscillatory behaviour is seen during sleep mode and it appears to be generated by a general brain synchronized state rather than specific synaptic activity.

\section{Future work}

The next step would be to model the cuneate interneurons as well as to construct cuneate circuitry models. These will be a theoretical framework for posing questions and predicting possible behaviours related to three important research lines:

(1) the local processing of somesthetic information in the $\mathrm{CN}$,

(2) the modulation exerted by the sensorimotor cortex on the local processing carried out by the cuneate and

(3) the participation of this nucleus in sleep rhythms.

With regard to the local processing, there exist data showing both excitatory and inhibitory recurrent effects $[5,36]$. With regard to the cortical modulation, it has been observed that the cerebral cortex is able to activate and/or inhibit both cuneate projection neurons and interneurons [4, 33, 34, 36]. Finally, recent discoveries [33-35, 37] have shown that the cuneate is capable of intrinsically generating delta rhythms (1-4 Hz) and of showing slower rhythms, these being induced by the cerebral cortex. If we integrate and match our model with all the experimental data, we will have taken a decisive step forward in the understanding of this part of the nervous system.

\section{Acknowledgments}

This work was supported by a grant from the DGICYT (PB96-0958). We would also like to thank the Laboratorios de Neurociencia y Computación Neuronal (LANCON), the environment in which this work has been developed.

\section{Appendix. Mathematical expressions for ionic currents}

- Sodium current $I_{\mathrm{Na}}$

$$
\begin{aligned}
I_{\mathrm{Na}} & =g_{\mathrm{Na}}^{\max } m_{\mathrm{Na}}^{3} h_{\mathrm{Na}}\left(V-V_{\mathrm{Na}}\right) & & \\
\alpha_{m} & =\frac{1.28(V+38)}{1-\exp [-(V+38) / 5]} & \beta_{m} & =\frac{-1.24(V+38)}{1-\exp [-(V+38) / 5]} \\
\alpha_{h} & =0.32 \exp \left(\frac{-(V+55)}{15}\right) & \beta_{h} & =\frac{41.4}{1+\exp [-(V-17) / 21]} .
\end{aligned}
$$


- Potassium current $I_{\mathrm{K}}$

$$
\begin{aligned}
I_{\mathrm{K}} & =g_{\mathrm{K}}^{\max } m_{\mathrm{K}}^{4}\left(V-V_{\mathrm{K}}\right) \\
\alpha_{m} & =\frac{0.1(-45-V)}{\exp [(-45-V) / 5]-1} \quad \beta_{m}=1.7 \exp \left(\frac{(-50-V)}{40}\right) .
\end{aligned}
$$

- High-threshold calcium current $I_{L}$

$$
\begin{aligned}
I_{L} & =g_{L}^{\max } m_{L}\left(V-V_{\mathrm{Ca}}\right) \\
\alpha_{m} & =\frac{16}{1+\exp [-(V+30)]} \quad \beta_{m}=\frac{0.004(V-1.31)}{\exp [(V-1.31) / 5.36]-1} .
\end{aligned}
$$

- Low-threshold calcium current $I_{T}$

$$
\begin{aligned}
I_{T} & =g_{T}^{\max } m_{T}^{2} h_{T}\left(V-V_{\mathrm{Ca}}\right) \\
m_{\infty} & =\frac{1}{1+\exp [-(V+52) / 6.2]} \\
\tau_{m} & =0.0025\left(\frac{1}{\exp [-(V+132) / 16.7]+\exp [(V+16.8) / 18.2]}+0.612\right) \\
h_{\infty} & =\frac{1}{1+\exp [(V+75) / 4]} \quad \tau_{h}=1 .
\end{aligned}
$$

- Calcium-dependent potassium current $I_{a h p}$

$$
\begin{aligned}
& I_{a h p}=g_{a h p}^{\max } m_{a h p}^{2}\left(V-V_{\mathrm{K}}\right) \\
& \alpha_{m}=10^{8}[\mathrm{Ca}]^{2} \quad \beta_{m}=10 .
\end{aligned}
$$

- Hyperpolarization-activated cationic current $I_{h}$

$$
\begin{aligned}
I_{h} & =g_{h}^{\max } m_{h}\left(V-V_{h}\right) \\
m_{\infty} & =\frac{1}{1+(V+75) / 5} \\
\tau_{m} & =\frac{1}{\exp [-14.59-(0.086 V)]+\exp [-1.87+(0.007 V)]} .
\end{aligned}
$$

\section{References}

[1] Abbott L F 1994 Single neuron dynamics: an introduction Neural Modeling and Neural Networks ed F Ventriglia (Oxford: Pergamon) pp 57-78

[2] Amassian V E and Griblin D 1974 Periodic components in steady-state activity of cuneate neurons and their possible role in sensory coding J. Physiol. Lond. 243 353-85

[3] Berkley K J, Badell R J, Blomqvist A and Bull M 1986 Output systems of the dorsal column nuclei in the cat Brain Res. Rev. 11 199-225

[4] Canedo A 1997 Primary motor cortex influences on the descending and ascending systems Prog. Neurobiol. 51 287-335

[5] Canedo A, Martínez L and Mariño J 1998 Tonic and bursting activity in the cuneate nucleus of the chloralose anesthetized cat Neuroscience 84 603-17

[6] Canedo A and Aguilar J 2000 Spatial and cortical influences exerted on cuneothalamic and thalamocortical neurons of the cat Eur. J. Neuroscience 12 2515-33

[7] Canedo A, Mariño J and Aguilar J 2000 Lemniscal recurrent and transcortical influences on cuneate neurons Neuroscience 97 317-34

[8] Cheema S, Fyffe R, Light A and Rustioni A 1984 Arborizations of single corticofugal axons in the feline cuneate nucleus stained by iontophoretic injection of horseradish peroxidase Brain Res. 290 158-64

[9] Coulter D A, Huguenard J R and Prince D A 1989 Calcium currents in rat thalamocortical relay neurons: kinetic properties of the transient low-threshold current J. Physiol. Lond. 414 587-604 
[10] Destexhe A, Contreras D, Sejnowski T J and Steriade M 1994 A model of spindle rhythmicity in the isolated thalamic reticular nucleus J. Neurophysiol. 72 803-18

[11] Deuchars S A, Trippenbach T and Spyer K M 2000 Dorsal column nuclei neurons recorded in a brain stem-spinal cord preparation: characteristics and their responses to dorsal root stimulation J. Neurophysiol. 84 1361-8

[12] Ermentrout G B 1990 PHASEPLANE: the Dynamical Systems Tool version 3.0 (Pacific Groove, CA: BrooksCole)

[13] Fyffe R E, Cheema S S and Rustioni A 1986 Intracelular staining study of the feline cuneate nucleus: I. Terminal patterns of primary afferent fibers $J$. Neurophysiol. 56 1268-83

[14] Gear C W 1967 The numerical integration of ordinary differential equations Math. Comput. 21 146-56

[15] Gordon G and Jukes M G M 1964 Descending influences on the exteroceptive organizations of the cat's gracile nucleus J. Physiol. Lond. 173 291-319

[16] Hines M 1989 A program for simulation of nerve equations with branching geometries Int. J. Bio-Med. Comput. 24 55-68

[17] Hodgkin A and Huxley A 1952 The components of membrane conductance in the giant axon of Loligo J. Physiol. Lond. 116 473-96

[18] Hodgkin A and Huxley A 1952 Currents carried by sodium and potassium ions through the membrane of the giant axon of Loligo J. Physiol. Lond. 116 449-72

[19] Hodgkin A and Huxley A 1952 The dual effect of membrane potential on sodium conductance in the giant axon of Loligo J. Physiol. Lond. 116 497-506

[20] Hodgkin A and Huxley A 1952 A quantitative description of membrane current and its application to conduction and excitation in nerve $J$. Neurophysiol. 117 500-44

[21] Hodgkin A, Huxley A and Katz B 1952 Measurements of current-voltage relations in the membrane of the giant axon of Loligo J. Physiol. Lond. 116 424-48

[22] Huguenard J R, Coulter D A and Prince D A 1988 Developmental changes in $\mathrm{Na}^{+}$conductances in rat neocortical neurons: appearance of a slowly inactivating component J. Neurophysiol. 59 778-95

[23] Huguenard J R, Coulter D A and Prince D A 1991 A fast transient potassium current in thalamic relay neurons: kinetics of activation and inactivation J. Neurophysiol. 66 1304-15

[24] Huguenard J R and McCormick D A 1992 Simulation of the currents involved in rhythmic oscillations in thalamic relay neurons J. Neurophysiol. $681373-83$

[25] Huguenard J R and Prince D A 1991 Slow inactivation of a TEA-sensitive K current in acutely isolated rat thalamic relay neurons J. Neurophysiol. 66 1316-28

[26] Jack J J B and Redman S J 1971 The propagation of transient potentials in some linear cable structures J. Physiol. Lond. 215 283-320

[27] Jahnsen H and Llinás R 1984 Electrophysiological properties of guinea-pig thalamic neurons: an in vitro study J. Physiol. Lond. 349 205-26

[28] Jahnsen H and Llinás R 1984 Ionic basis for the electroresponsiveness and oscillatory properties of guinea-pig thalamic neurons in vitro J. Physiol. Lond. 349 227-47

[29] Kay A R and Wong R K S 1987 Calcium current activation kinetics in isolated pyramidal neurons of the CA1 region of the mature guinea-pig hippocampus J. Physiol. Lond. 392 603-16

[30] Kuypers H G J M and Tuerk J D 1964 The distribution of the cortical fibres within the nuclei cuneatus and gracilis in the cat J. Anat. 98 143-62

[31] Lisman J E 1997 Bursts as a unit of neural information: making unreliable synapses reliable Trends Neurosci. 20 38-43

[32] Lytton W W, Destexhe A and Sejnowski T J 1996 Control of slow oscillations in the thalamocortical neuron: a computer model Neuroscience 70 673-84

[33] Mariño J 1998 Influencia de la corteza sensoriomotora sobre la transmisión somatosensorial en el gato anestesiado PhD Thesis Universidad de Santiago de Compostela

[34] Mariño J, Canedo A and Aguilar J 2000 Sensorimotor cortical influences on cuneate nucleus rhythmic activity in the anesthetized cat Neuroscience 95 657-73

[35] Mariño J, Aguilar J and Canedo A 1999 Cortico-subcortical synchronization in the chloralose-anesthetized cat Neuroscience 93 409-11

[36] Mariño J, Martínez L and Canedo A 1999 Sensorimotor integration at the dorsal column nuclei News Physiol. Sci. 14 231-7

[37] Mariño J, Martínez L and Canedo A 1996 Coupled slow and delta oscillations between cuneothalamic and thalamocortical neurons in the chloralose anesthetized cat Neurosci. Lett. 219 107-10

[38] Martínez L 1997 Fibras pericruciadas con colaterales al núcleo rojo, a la formación reticular medial bulbar y a los núcleos de las columnas dorsales. Efectos sinápticos de las células del tracto piramidal sobre las neuronas del núcleo cuneatus PhD Thesis Universidad de Santiago de Compostela 
[39] Martínez L, Lamas J A and Canedo A 1995 Pyramidal tract and corticospinal neurons with branching axons to the dorsal column nuclei of the cat Neuroscience 68 195-206

[40] McCormick D A and Bal T 1997 Sleep and arousal: thalamocortical mechanisms Ann. Rev. Neurosci. 20 185-215

[41] McCormick D A and Huguenard J R 1992 A model of the electrophysiological properties of thalamocortical relay neurons J. Neurophysiol. 68 1384-400

[42] McCormick D A and Pape H C 1990 Noradrenergic and serotoninergic modulation of a hyperpolarizationactivated cation current in thalamic relay neurons J. Physiol. Lond. 431 319-42

[43] McCormick D A and Pape H C 1990 Properties of a hyperpolarization-activated cation current and its rate in rhythmic oscillations in thalamic relay neurons J. Physiol. Lond. 431 291-318

[44] Nuñez A and Buño W 1999 In vitro electrophysiological properties of rat dorsal column nuclei neurons Eur. J. Neurosci. 11 1865-76

[45] Rall W 1964 Theoretical significance of dendritic tree for input-output relation Neural Theory and Modeling ed R F Reiss (Stanford, CA: Stanford University Press) pp 73-97

[46] Reboreda A, Sánchez E, Romero M and Lamas J A 2002 Spontaneous firing and subthreshold oscillation in neurones of the rat dorsal column nuclei in culture FENS Forum Abstr. p 544

[47] Sherman S and Koch C 1990 Thalamus The Synaptic Organization of the Brain ed G M Shepherd (New York: Oxford University Press) pp 246-78

[48] Sánchez E, Barro S, Canedo A, Martínez L and Mariño J 1998 A computational model of cuneate nucleus interneurons Eur. J. Neurosci. 10402 (abstract)

[49] Soltesz I, Lightowler S, Leresche N, Janik-Gerschenfeld D, Pollard C E and Crunelli V 1991 Two inward currents and the transformation of low frequency oscillations of rat and cat thalamocortical cells J. Physiol. Lond. 441 175-97

[50] Sotgiu M L and Margnelli M 1976 Electrophysiological identification of pontomedullary reticular neurons directly projecting into dorsal column nuclei Brain Res. $103443-53$

[51] Steriade M and LLinás R 1988 The functional states of the thalamus and the associated neuronal interplay Phys. Rev. 68 649-742

[52] Steriade M, McCormick D A and Sejnowski T J 1993 Thalamocortical oscillations in the sleeping and aroused brain Science 262 679-85

[53] Steriade M 2001 Impact of network activities on neuronal properties in corticothalamic systems J. Neurophysiol. $861-39$

[54] Traub R D and Wong R K S 1982 Cellular mechanism of neuronal synchronization in epilepsy Science 216 $745-7$

[55] Wang X J, Rinzel J and Rogawski M A 1991 A model of the T-type calcium current and the low threshold spike in thalamic neurons J. Neurophysiol. 66 835-9

[56] Yamada W M, Koch C and Adams P R 1989 Multiple channels and calcium dynamics Methods in Neuronal Modeling ed C Koch and I Segev (New York: MIT Press) pp 20-45 